\title{
JAPANESE TRANSLATION OF THE PROSTHETIC LIMB USERS SURVEY OF MOBILITY
}

\author{
Geoffrey Balkman*, Soshi Samejima, David Aoki, Brian J. Hafner \\ Department of Rehabilitation Medicine, University of Washington, Seattle, WA, USA. \\ *Email: gbalkman@uw.edu
}

DOI: https://doi.org/10.33137/cpoj.v1i2.32017

\section{INTRODUCTION}

Patient-reported outcome measures (PROMs) are used by prosthetists to assess and communicate effectiveness of prosthetic interventions. The Prosthetic Limb Users Survey of Mobility (PLUS-M) is a PROM developed to evaluate lower limb prosthetic mobility. ${ }^{1}$ Valid and reliable translations of prosthetics-specific PROMs, like PLUS-M, do not exist for Japanese-speaking patients. The purpose of this research was to translate PLUS-M to Japanese using rigorous translation guidelines in order to maintain the meaning and applicability of items across both languages.

\section{METHODS}

Translation efforts followed recommended processes and best practices. ${ }^{2}$ First, PLUS-M items $(n=44)$, response options and instructions were independently translated by investigators from English to Japanese. Translators met with the principal PLUS-M developer to discuss the forward translations and resolve any discrepancies. Reconciliation guidelines ${ }^{3}$ were used to code and document decisions. The reconciled translation was back-translated from Japanese to English by a bilingual collaborator. Investigators compared the back-translation relative to the English instrument, and the developer identified items that required modification. All translations and revisions were recorded in a comprehensive translation document. Four expert reviewers in Japan evaluated the translation and provided additional suggestions for revisions.

\section{RESULTS}

Twenty-one items had to be removed or revised in the translation process. One item without a comparable situational context was removed during forward translation. The PLUS-M item that described shag (highpile) carpet was removed because that type of carpet is not common in Japan. Seventeen items were revised grammatically to maintain the intended purposes of the original English items. For example, a PLUS-M item that asks respondents how well they can "step off" an escalator was back-translated as how well one could "get off" the escalator. The investigators added additional terms to the translation (i.e., "get off an escalator step over step") to ensure the item was read and interpreted as intended. More substantial revisions were made to three items to address cultural or environmental differences. For example, the expert reviewers noted that the term "block" in the PLUS-M item, "Are you able to walk a block on flat ground?" does not translate to Japanese. The investigators, in consultation with the expert reviewers, suggested the phrases "from one traffic light to the next," "50 - 100 meters," or "from one intersection to the next," could be used as alternatives. The developer preferred the phrase with "intersection." This revision changed the original item, but was understandable in Japanese and aligned with the developers' intended meaning (i.e., walking moderate distances outdoors). The final translated items were used to create 7- and 12-item short forms suited to administration in clinics or research studies (Figure 1).

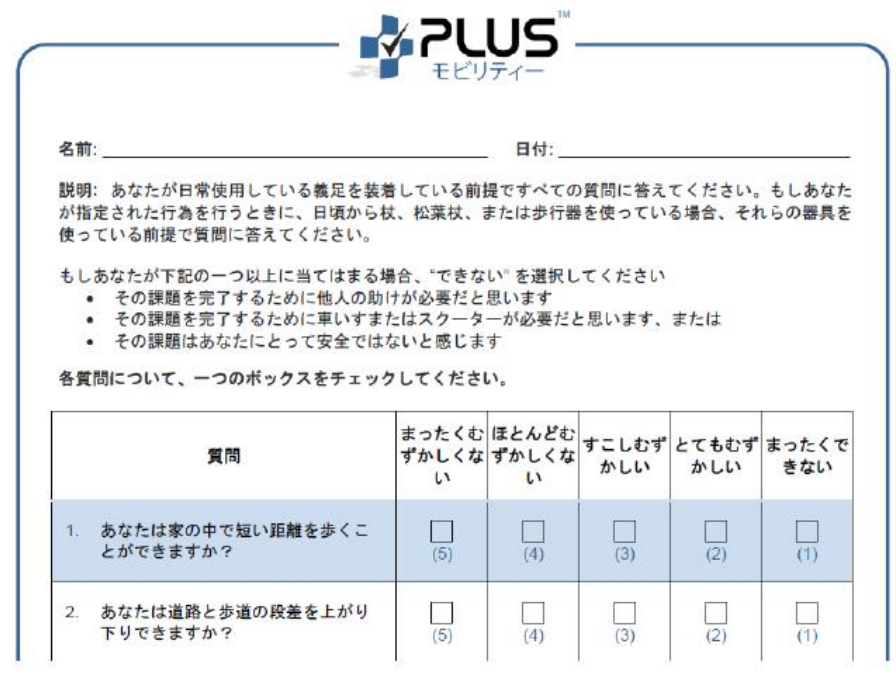

Figure 1. Japanese translation of PLUS-M short form 


\section{CONCLUSION}

The PLUS-M Japanese version is ready for testing by prosthetics experts in Japan. Cognitive interviews with Japanese prosthesis users will assess the clarity and comprehensiveness of items. Large-scale administration and comparison to US norms will be conducted to quantitatively assess equivalence between the original and the Japanese translation of PLUS-M .

\section{SIGNIFICANCE}

International and cross-cultural comparisons of key health outcomes, like mobility, can help to assess the relative effectiveness of prosthetic interventions. Use of standardized outcome measures, like PLUS-M, can facilitate comparisons and improve the standard of care worldwide.

\section{REFERENCES}

1. Hafner BJ, et al. Construct validity of the Prosthetic Limb Users Survey of Mobility (PLUS-M) in adults with lower limb amputation. Arch Phys Med Rehabil. 2017;98(2):277- 5. DOI:10.1016/j.apmr.2016.07.026

2. Eremenco SL, et al. A comprehensive method for the translation and cross-cultural validation of health status questionnaires. Eval Health Prof. 2005;28(2):212-32. DOI: $\underline{10.1177 / 0163278705275342}$

3. Koller M, et al. The process of reconciliation: evaluation of guidelines for translating quality-of-life questionnaires. Expert Rev Pharmacoecon Outcomes Res. 2012;12(2):189-97. DOI:10.1586/erp.11.102

\section{DISCLOSURE}

There is no current or pending support pertaining to this project.

\section{ACKNOWLEDGEMENTS}

The authors thank Toshiki Kobayashi, PhD, Kazuhiro Sakai, Masanori Shimamura CPO, and Noriaki Maeda, $\mathrm{PhD}$ for their review of the PLUS-M Japanese translation. 